\title{
Specific IgA antibodies in Mycoplasma pneumoniae infections
}

\author{
Gino Ciarrocchi', Massimo Tocchini', Marco d'Anzeo', Elisabetta Farnocchia', Giuseppe \\ Rondello', Maria Enrica Cimarelli ${ }^{2}$, Brunilde Berti ${ }^{3}$, Andrea lanniello ${ }^{3}$
}

I S.O. Laboratorio analisi, Sierologia, A.O. Ospedali Riuniti, Ancona;

2 S.O. Broncopneumologia, A.O. Ospedale Civile, Jesi;

3 Centro Ricerca e Sviluppo, Diesse, Siena

Key words: Antibodies, ELISA, CAP

\section{Anticorpi IgA nelle infezioni da Mycoplasma pneumoniae}

\section{SUMMARY}

Mycoplasma pneumoniae is the prevalent cause of community acquired pneumonia (CAP), especially in pediatric age. M. pneumoniae infection may presents a severe pulmonary disease whose clinical picture is undistinguishable from that of other pneumotropic agents. A rapid and suitable laboratory diagnosis mainly lies on serological test investigations. A total of 37 consecutive CAP pediatric and adult patients, and 102 subjets with suspected respiratory tract infections (SIR) were enrolled and examined for specific IgA-, IgG-, IgM anti-M. pneumoniae antibodies using a rapid ELISA test (Mycoplasma Chorus-Diesse), and a two step ELISA test (MYP test-Savyon). Agreement of IgA, IgG, and IgM values were $96 \%, 64 \%, 100 \%$, respectively.

Among CAP patients, IgM was the prevalent marker (23/37); in this population, 12/23 pediatric patients were both IgM and IgA positives. Conversely, among adults, IgA antibodies were the most prevalent infection marker. Pediatric patients of both CAP and SIR population showed a similar serological picture; instead, all adult patients resulted as IgM negatives; IgA and IgG antibodies showed high positive values in $4 / 8$, and in four old patients IgA was the alone infection marker.

Although specific lgM antibodies detection remains the main serological sign of recent infection in pediatric age, the presence of $\lg A$ antibodies is a suitable tool to revealing the etiology of clinical picture in both pediatric and adult patients.

\section{INTRODUZIONE}

Mycoplasma pneumoniae è uno dei principali agenti di polmonite atipica acquisita in comunità (CAP) e di infezioni delle alte e basse vie respiratorie, prevalente in età pediatrica. Il ruolo rilevante di M. pneumoniae nelle esacerbazioni di bronchiti croniche ostruttive (COPD) e in manifestazioni extrapolmonari è stato ampiamente dimostrato $(3,7,9,11,12,17,18)$. A causa della genericità dei sintomi, comuni a quelli provocati da altri agenti patogeni, è necessaria una dirimente diagnosi eziologica, poiché un trattamento dell'infezione con antibiotici beta-lattamici risulterebbe incongruo; viceversa, l'impiego di macrolidi o tetracicline ha positivi effetti sulla durata e risoluzione della malattia $(11,13)$.

Particolarmente suscettibili a forme infettive acute sono i soggetti in età pediatrica, mentre nell'adulto sono più frequenti le reinfezioni con ampia variabilità dei quadri clinici $(10,15)$. In assenza di un gold standard microbiologico, la diagnosi di laboratorio riveste grande rilevanza nel contesto clinico, potendo contare su test di differente efficienza e rapidità. La ricerca di anticorpi specifici su campioni di siero è quella maggiormente perseguita. La tecnica immunoenzimatica (ELISA) ha in gran parte sostituito la tradizionale Reazione di Fissazione del Complemento (RFC); l'esame colturale è di modesto impiego nella diagnostica clinica a causa dei lunghi tempi di risposta e della bassa sensibilità $(2,14)$; l'introduzione della metodologia di amplificazione genica (PCR) su campioni del tratto respiratorio dimostra sensibilità e specificità elevate, ma non differenzia tuttavia la colonizzazione dall'infezione $(3,4,6)$. La tecnica ELISA è attualmente il metodo più diffuso, poiché permette di ottenere un pattern sierologico specifico mediante la distinta rilevazione quantitativa degli isotipi $\operatorname{IgA}, \operatorname{IgG}, \operatorname{IgM}(1,3,8$, 10, 11, 19). Essi hanno differente significato e prevalenza: infezioni correnti o recenti sono caratterizzate dalla produzione di IgM e, spesso, IgA, associati a IgG, in relazione allo stato dell'infezione. Il significato di IgA non è stato ben messo in

\section{Corresponding author: Gino Ciarrocchi}

S.O. Laboratorio analisi, Sierologia, A.O. Ospedali Riuniti - Ancona

Tel.: 07I-596425I - Fax: 07I-5964638

E-mail: ciarrokki@libero.it 
luce, con verosimile sottostima dell'efficacia diagnostica, fatta eccezione della sua rilevanza nei casi di esacerbazione di COPD, poiché anticorpi IgA compaiono più precocemente di IgM e decrescono più rapidamente $(2,3,5,7,10,13,17)$. L'utilizzo di un test ELISA con elevata efficienza è fondamentale per rivelare l'eziologia dell'infezione; viceversa, l'impiego di test basati su antigeni non selettivi, ricchi di frazioni glicolipidiche conduce a risultati talora fuorvianti, con negative ricadute sulla decisione terapeutica $(2,16,19)$. Infine, la determinazione di un pattern sierologico completo nelle infezioni da $M$. pneumoniae permette di comprendere l'andamento della risposta anticorpale, in relazione ai differenti quadri clinici espressi $(5,13,16)$.

Lo scopo dello studio è quello di valutare il significato di anticorpi IgA anti- Mycoplasma pneumoniae in pazienti pediatrici e adulti, determinati con un test ELISA rapido.

\section{MATERIALI E METODI}

Un totale di 139 campioni di siero raccolti da pazienti adulti e pediatrici sono stati esaminati per la ricerca di anticorpi di classe $\operatorname{IgA}, \operatorname{IgG}, \operatorname{IgM}$ antiM. pneumoniae. I pazienti sono stati clinicamente classificati in due gruppi: i) 37 soggetti adulti e pediatrici ricoverati con quadro clinico di polmonite atipica acquisita in comunità (CAP); ii) 102 soggetti con sospetta infezione delle alte e basse vie respiratorie (SIR). I soggetti di ciascun gruppo furono suddivisi secondo fasce di età 1-20, 21-40, 41-80 anni. I sieri sono stati processati con tre distinti test ELISA per la ricerca rapida di IgA, IgG, IgM (Mycoplasma Chorus - Diesse Diagnostici, Siena), eseguiti con singolo dispositivo in completa automazione tramite un mini analizzatore a rotore circolare (Chorus analyzer Diesse Diagnostici, Siena). I test Chorus IgA e IgM impiegano un antigene derivato da M. pneumoniae (ceppo M129), costituito da un estratto purificato per sonicazione, doppio trattamento con detergente e centrifugazione, per ottenere un'elevata concentrazione di proteina P1 (adesina); il test IgG impiega un estratto intero ottenuto da coltura di M. pneumoniae (ceppo M129), trattato con detergente e centrifugato. Il test di confronto adotta il metodo ELISA classico (MYP-test IgA, IgG, IgM - Savyon, Israele; Eurospital, Trieste), con impiego di uno stesso coating antigenico per ogni singolo test, costituito da un estratto purificato di $M$. pneumoniae (ceppo M129), arricchito di proteina P1. Nei 37 pazienti CAP, il confronto tra i due test è stato effettuato per completezza sulle tre classi anticorpali, mentre nel gruppo dei 102 pazienti SIR il confronto è stato limitato a IgA, oggetto dello studio.

\section{RISULTATI}

La Tabella 1 illustra il numero dei pazienti esaminati, suddivisi in fasce di età all'interno di ogni gruppo di patologia: 37 pazienti CAP e 102 pazienti SIR.

Tra i 37 pazienti CAP, i test Chorus M. pneumoniae $\operatorname{IgA}, \mathrm{IgG}, \mathrm{IgM}$ mostrarono una concordanza totale con i corrispondenti test Savyon, rispettivamente di 96\%, 64\%, 100\%, (Tabella 2). Nei 102 pazienti SIR la concordanza per IgA fu del 93.1\% (Tabella 3).

La prevalenza delle differenti classi anticorpali, il singolo riscontro o la loro contestuale associazione, nel gruppo di pazienti CAP, sono illustrati nella Tabella 4, secondo le fasce di età. Nel totale, anticorpi IgM risultarono il marcatore sierologico più rilevante (30/37); anticorpi IgM erano associati a $\operatorname{IgA}$ in 12 casi, nella fascia di pazienti pediatrici (età 1-20); viceversa, nella fascia di adulti-anziani (età 41-80), anticorpi IgA hanno il maggior riscontro, associati in prevalenza a $\mathrm{IgG}$ $(5 / 9)$.

Un analogo andamento si osserva nella popolazione di 102 pazienti SIR: anticorpi IgA erano associati a IgM in 10/28, nella fascia di età 1-20; nella fascia di pazienti adulti-anziani (41-80), anticorpi IgA non erano mai associati a IgM, mentre risultarono positivi, associati a $\operatorname{IgG}$ in 4 pazienti, in altri quattro casi IgA rappresentavano l'unico marcatore d'infezione (Tabella 5).

\section{DISCUSSIONE}

L'indagine sierologica delle infezioni da Mycoplasma pneumoniae rappresenta un presidio utile alla definizione del quadro clinico-diagnostico, ma può anche diventare un rompicapo fuorviante, se i test impiegati hanno una mediocre efficienza diagnostica. La determinazione distinta di classi anticorpali specifiche, con impiego di coating antigenici selettivi delinea, viceversa, un pattern sierologico significativo dello stato dell'infezione, riducendo cross-reazioni dovute alle componenti glicolipidiche del germe, condivise da altri batteri $(2,16)$. Ciò consente di ottenere una specifica diagnosi eziologica, con positive ricadute sulla rapidità e congruità delle strategie terapeutiche da adottare.

Nello studio, anticorpi di classe IgA anti-M. pneumoniae sono stati determinati, unitamente a $\operatorname{IgM}$ e $\mathrm{IgG}$, attraverso il confronto tra due test con analoghe e selettive caratteristiche antigeniche e metodologiche. La concordanza è risultata eccellente sia per la ricerca di IgA che per IgM; le discordanze tra i due test IgG sono verosimilmente riconducibili alla differente natura del coating antigenico impiegato. Alla dimostrata affidabilità del test ELISA rapido si può aggiungere la capacità di for- 
nire un completo e specifico quadro sierologico, con forte riduzione dei tempi di referto, rispetto al test ELISA classico.

Come altrove dimostrato $(2,3,10)$, un riscontro significativo di anticorpi IgM viene associato a casi di infezione recente o in atto, specialmente nelle fasce di età pediatrica e giovanile, pur dovendo considerare che l'isotipo IgM mostra sovente un lento declino; la nostra esperienza dimostra che in tali pazienti anticorpi IgM sono associati spesso a valori elevati di $\operatorname{IgA}$, il cui significato può essere assunto quale marcatore immunologico di un'infezione on-going.

Viceversa, nell'età adulta e specialmente nell'anziano anticorpi IgM sono molto meno rilevabili, come ben evidenziato nei due gruppi di pazienti in studio; in tali popolazioni l'infezione acuta o la reinfezione sono espresse prevalentemente da elevati valori di $\operatorname{IgA}$, in presenza di $\mathrm{IgG}$. È stato dimostrato altresì, in quattro pazienti anziani SIR, un significativo riscontro di IgA anti-M. pneumoniae quale unico marcatore d'infezione.

Nello studio non compaiono casi di soggetti con patologie da $\mathrm{BCPO}$, in cui, come ampiamente dimostrato in letteratura, anticorpi IgA antiMycoplasma pneumoniae costituiscono il marcatore infettivo più rilevante nelle esacerbazioni della malattia (7); tuttavia, anche in quadri clinici di CAP o di sospette infezioni respiratorie in pazienti adulti-anziani, la presenza di anticorpi IgA contribui-

Tabella I. Popolazioni di pazienti studiati suddivisi per fasce di età.

\begin{tabular}{cccc}
\hline Fasce di età & Pazienti SIR & Pazienti CAP & Totale pazienti \\
\hline$I-20$ & 44 & 23 & 67 \\
\hline $2 \mid-40$ & $2 \mid$ & 4 & 25 \\
\hline $4 \mid-80$ & 37 & 10 & 47 \\
\hline Totale & 102 & 37 & 139 \\
\hline
\end{tabular}

Tabella 2. Confronto tra Mycoplasma pneumoniae $\lg A, \lg G, \lg M$ Chorus e Mycoplasma pneumoniae $\lg A$, $\lg G, \lg M$ Savyon in 37 pazienti CAP.

\begin{tabular}{ccccc}
\hline Test & positivo & border-line & negativo & concordanza \\
\hline Chorus IgA & 22 & 2 & 13 & $96 \%$ \\
Savyon IgA & 17 & 8 & 12 & \\
\hline Chorus IgM & 30 & 0 & 7 & $100 \%$ \\
Savyon IgM & 25 & 5 & 7 & $64 \%$ \\
\hline Chorus IgG* & 36 & 0 & 1 & \\
Savyon $\lg G^{* *}$ & 12 & 11 & 14 & \\
\hline
\end{tabular}

*coating estratto intero; **coating Proteina PI

Tabella 3. Confronto tra Mycoplasma pneumoniae IgA Chorus e Mycoplasma pneumoniae IgA Savyon in 102 pazienti SIR.

\begin{tabular}{ccccc}
\hline Test & positivo & border-line & negativo & concordanza \\
\hline Chorus IgA & 15 & 12 & 75 & \\
Savyon IgA & 14 & 6 & 82 & $93,1 \%$ \\
\hline
\end{tabular}

Tabella 4. Distribuzione di lgA-, lgG-, IgM anti- Mycoplasma pneumoniae in 37 pazienti CAP distinti in fasce d'età.

\begin{tabular}{ccccccc}
\hline Età & $N^{\circ}$ pazienti & IgM totali & $\lg A$ totali & $\lg M+\lg A$ & $\lg M+\lg G$ & $\lg A+\lg G$ \\
\hline $\mathrm{I}-20$ & 23 & 23 & 12 & 12 & 10 & 0 \\
\hline $2 \mathrm{I}-40$ & 4 & 3 & 3 & 2 & 1 & 1 \\
\hline $4 \mathrm{I}-80$ & 10 & 4 & 9 & 4 & 0 & 5 \\
\hline Totale & 37 & 30 & 24 & 18 & 11 & 6 \\
\hline
\end{tabular}

Tabella 5. Distribuzione di IgA-, IgG-, IgM anti- Mycoplasma pneumoniae in 102 pazienti SIR distinti in fasce d'età

\begin{tabular}{cccccccc}
\hline Età & $N^{\circ}$ pazienti & $\lg M$ totali & $\lg A$ totali & $\lg M+\lg A$ & $\lg M+\lg G$ & $\lg A+\lg G$ & $\lg A$ \\
\hline $0-20$ & 44 & 28 & 10 & 10 & 4 & 0 & 0 \\
\hline $21-40$ & 21 & 10 & 9 & 6 & 2 & 3 & 0 \\
\hline $4 I-80$ & 37 & 4 & 8 & 0 & 0 & 4 & 4 \\
\hline Totale & 102 & 42 & 27 & 16 & 6 & 7 & 4 \\
\hline
\end{tabular}


scono a definire il quadro diagnostico.

In conclusione, lo studio mette in evidenza, oltre ai ben noti caratteri di precocità di comparsa e di breve emivita, una caratteristica ambivalenza immunologica degli anticorpi IgA nelle infezioni da M. pneumoniae, in grado di delineare un significativo pattern sierologico sia nelle infezioni primarie pediatriche che nelle reinfezioni dell'adulto; inoltre, la possibilità di impiegare test selettivi e di rapida esecuzione ne rafforza ulteriormente il ruolo diagnostico e dirimente nelle infezioni respiratorie causate da altri agenti eziologici atipici.

\section{BIBLIOGRAFIA}

1. de Barbeyrac B, Obeniche F, Ratsima E, et al. Serological diagnosis of chlamydial and Mycoplasma pneumoniae infections. Ann Biol Clin (Paris) 2006; 64: 409-19.

2. Beersma MFC, Dorven K, van Dam AP, Templeton $\mathrm{Ke}$, Class ECJ, Goossens H. Evaluation of 12 commercial tests and the complement fixation test for Mycoplasma pneumoniae-specific immunoglobulin $\mathrm{G}$ (IgG) and IgM antibodies, with PCR used as the "gold standard". J Clin Microbiol 2005; 43(5): 2277-85.

3. Csàngò $\mathrm{PA}$, Pedersen JE, Hess RD. Comparison of four Mycoplasma pneumoniae IgM-, IgG- and IgAspecific enzyme immunoassays in blood donors and patients. Clin Microbiol Infect 2004; 10: 1094-8.

4. Dorigo-Zetsma JW, Zaat SAJ, Wertheim-van Dillem PME, et al. Comparison of PCR, culture, and serological test for diagnosis of Mycoplasma pneumoniae respiratory tract infection in children. J Clin Microbiol 1999; 37: 14-7.

5. Granström M, Holme T, Sjögren AM, Örtqvist A, Kalin $\mathrm{M}$. The role of IgA determination bey ELISA in the early serodiagnosis of Mycoplasma pneumoniae infection, in relation to IgG and $\mu$-capture IgM methods. J Med Microbiol 1994; 40: 288-92.

6. Lee EY, Lee DJ, Kim SW, Chang MW. Comparison of PCR, culture and serological tests for diagnosis of Mycoplasma pneumoniae infection. Pediatric Allergy Respir Dis, 2005; 15: 359-67.

7. Lieberman D, Lieberman D, Ben-Yaakov M, et al. Serological evidence of Mycoplasma pneumoniae infection, in acute exacerbation of COPD. Diagn Microbiol Infect Dis, 2002; 44: 1-6.
8. Liu FC, Chen PY, Huang FL, Tsai CR, Lee CY, Lin CF. Do serological tests provide adequate rapid diagnosis of Mycoplasma pneumoniae infection? Jpn J Infect Dis, 2008; 61: 397-9.

9. Narita M. Pathogenesis of extrapulmonary manifestations of Mycoplasma pneumoniae infection with special reference to pneumonia. J Infect Chemother, 2010 (Epub ahead of print).

10. Narita M. Evaluation of ELISA kits for detection of Mycoplasma pneumoniae-specific IgG, IgA, IgM antibodies on the diagnosis of Mycoplasma pneumoniae infection in children. Kansenshogaku Zasshi. 2005; 79: 457-63.

11. Schmidt-Ioanas M, Bender M, Roth A, Allewelt M, de Roux A, Lode H. Serologic early diagnosis of pneumonia caused by Mycoplasma pneumoniae. Dtsch Med Wochenschr. 2006; 131: 613-7.

12. Sliman JA, Metzgar D, David C, et al. Outbreak of acute respiratory disease caused by Mycoplasma pneumoniae on board a deployed U.S. Navy ship. $J$ Clin Microbiol, 2009; 47(12): 4121-3.

13. Soo Jin Yoo, Hye-Jeon Oh, Bo-Moon Shin. Evaluation of four commercial IgG- and IgM-specific enzyme immunoassays for detecting Mycoplasma pneumoniae antibody: comparison with particle agglutination assay. J Korean Med Sci, 2007; 22: 795-801.

14. Thacker WL, Talkington DF. Analysis of complement fixation and commercial enzyme immunoassays for detection of antibodies to Mycoplasma pneumoniae in human serum. Clin Diagn Lab Immunol, 2000; 7: 77880 .

15. Thurmin KA, Walter ND, Schwartz SB, et al. Comparison of laboratory diagnostic procedures for detection of Mycoplasma pneumoniae in community outbreaks. Clin Infect Dis, 2009; 48: 1244-9.

16. Tuuminen T, Suni J, Kleemola M, Jacobs E. Improved sensitivity and specificity of enzyme immunoassays with P1-adhesin enriched antigen to detect acute of Mycoplasma pneumoniae infection. $J$ Microbiol Methods, 2001; 44: 27-37.

17. Varma-Basil M, Dwivedi SK, Kumar K, et al. Role of Mycoplasma pneumoniae infection in acute exacerbation of chronic obstructive pulmonary disease. $J$ Med Microbiol, 2009; 58: 322-6.

18. Waites KB, Talkington DF. Mycoplasma pneumoniae and its role as a human pathogen. Clin Microbiol Rev, 2004; 17: 697-728.

19. Watkins-Riedel T, Stanek G, Daxboeck F. Comparison of SeroMP IgA with four other commercial assays for serodiagnosis of Mycoplasma pneumoniae pneumonia. Diagn Microbiol Infect Dis 2001; 40: 21-5. 